\title{
Neuropsychological findings in a non-clinical sample of workers exposed to solvents
}

\author{
I Reinvang, H M Borchgrevink, O Aaserud, V Lie, U F Malt, P Nakstad, P G Larsson, \\ L Gjerstad
}

\begin{abstract}
The risk of long-term damage to the CNS after exposure to mixed solvents in work environments is controversial. Thirty-six workers were studied who had been exposed to organic solvents for more than 10 years (mean 24.5 years) in a working environment. The workers and unexposed controls were studied with a battery of neuropsychological and cognitive tests. Significant group differences were observed for the Wechsler adult intelligence scale (WAIS) digit span and symbol digit substitution, and on paired associate learning and continuous word recognition. The results suggest that long-term work-related exposure to organic solvents may have chronic toxic effects.
\end{abstract}

$(\mathcal{F}$ Neurol Neurosurg Psychiatry 1994;57:614-616)

The risk of encephalopathy in occupational exposures to organic solvents is controversial, but positive findings have been reported in some studies. ${ }^{1-4}$ Further research must take into consideration several methodological problems. The use of self-referred groups may mean that high symptom levels and stress related to a failure to function in the work environment may have influenced the results in some studies. ${ }^{14}$ Mikkelsen et $a l^{3}$ point out that several studies have included workers with extremely short periods of exposure (less than one year), which are unlikely to give an increased risk of long-term effects. This study takes account of these criticisms and includes workers who were fully employed at the time of the study and with a history of more than 10 years of exposure.

The choice of behavioural variables needs to consider issues of sensitivity and specificity. Neuropsychological test batteries have been validated in relation to the prediction of structural brain damage in trauma and stroke and may not be sensitive to chronic toxic brain damage. Performance measures based on simple tasks and the precise measurement of reaction time ${ }^{5}$ have shown sensitivity to mild, acute toxic influences, ${ }^{6}$ but there is little information on how to interpret them clinically in patients with chronic disease. An approach combining neuropsychological and performance measures may have advantages.

This work is part of a larger project which is studying neurological, neuroradiological, and neurobehavioural findings in a group of workers exposed to mixed organic solvents for at least 10 years, and in types of work known to include a high level of exposure. We report the neuropsychological findings on a set of measures including clinical neuropsychological tests and performance measures based on reaction time.

\section{Subjects and methods METHODS}

Behavioural tests were chosen to be representative of earlier studies and recommendations from international review groups. The socalled WHO battery is recommended by an international review group to provide a common reference for cooperative studies. ${ }^{7}$ Tests belonging to this battery are marked (WHO) in the following.

Subtests from the Wechsler adult intelligence scale (WAIS) included similarities, block design, digit span (WHO), and digit symbol substitution (WHO). Memory tests included the paired associate learning test and symbol digit recall test used in previous neurotoxicological studies. ${ }^{18}$

Standard clinical neuropsychological tests ${ }^{9}$ included a non-verbal clinical memory test, Kimura recurring figures, with a recognition response. The trail-making test, parts $\mathrm{A}$ and $B$, was included as a measure of cognitive speed, and the grooved pegboard test as a measure of dexterity and coordination.

A continuous word recognition task ${ }^{10}$ was adapted for use in this study. A series of 90 words is shown on a computer screen with three-second intervals. Thirty words are shown only once and 30 words are shown twice. The number of words intervening between recurring words may be two, four, or six (short interval), or 12,24 , or 36 (long interval). The reaction time to yes/no buttonpress and accuracy is measured.

Reaction time (WHO) is a sensitive measure of acute neurobehavioural effects and is measured in this study with the simple reaction time and continuous performance tests from the NES 2 computerised battery. ${ }^{11}$ Tests of manual dexterity and coordination (WHO) include a computerised test, hand-eye coordination from NES 2 . The WHO battery includes a self-rating of mood and this study uses the version of profile of mood states from the NES 2 .

\section{SUBJECTS}

Thirty-six male workers exposed to organic solvents for more than 10 years in a working 
environment were studied. They were recruited from military sites on the basis of available exposure records of work tasks including construction painting, degreasing, or handling of jet fuel. All of the workers had been exposed to mixtures of solvents, mainly white spirit and various paints, and the same worker may have been exposed to different combinations of solvents at different times.

The workers were all fully employed at the time of the study, some of them still working with solvents, but with low exposure levels in recent years. None was seeking compensation for work-related injury at the time of entering the study. The mean (SD) age was $44.5(8 \cdot 0)$ years, and the mean (SD) length of exposure was $24.2(7 \cdot 5)$ years. Most $(80 \%)$ had an educational level of less than 12 years of general education with additional workrelated training.

Controls were selected as pairs matched for sex, age, and level of education from unexposed workers at the same work sites by the local medical officer. If a certain site had five workers satisfying the exposure inclusion criteria for the study, then the local medical officer also recruited five controls from the same site on a best-match basis. The resulting control group (mean (SD) age 44.1 (8.1) years) was well matched to the exposed group on demographic variables. The study group and controls were screened for medical history and alcohol consumption. Subjects were excluded if they had a history of head trauma with unconsciousness on admission to hospital, had other known brain disease, diabetes, or a history of drug or alcohol abuse.

Neuropsychological and other studies were performed blindly, without knowledge of the exposure status of the subjects, and the group membership code was not broken until data collection for the whole study was complete.

Table 1 Scores on WAIS scales for exposed and unexposed workers. Values are median $(Q 3-Q 1)$ scores

\begin{tabular}{lllll}
\hline Test & $\begin{array}{l}\text { Exposed } \\
(n=36)\end{array}$ & $\begin{array}{l}\text { Unexposed } \\
(n=36)\end{array}$ & $Z$ value & $p$ value \\
\hline Digit span-forward & $5(1)$ & $6(2)$ & 2.27 & 0.023 \\
Digit span-backward & $4(2)$ & $4.5(1)$ & 2.30 & 0.021 \\
Digit symbol & $38 \cdot 5(13.5)$ & $43.5(15)$ & 2.90 & 0.004 \\
Similarities & $17.5(6)$ & $18(6)$ & 0.96 & 0.33 \\
Block design & $37.5(11)$ & $37.5(14)$ & 0.60 & 0.55 \\
\hline
\end{tabular}

Table 2 Neuropsychological and performance test results in exposed and unexposed workers. Values are median $(Q 3-Q 1)$ scores

\begin{tabular}{|c|c|c|c|c|}
\hline Test/parameter & $\begin{array}{l}\text { Exposed } \\
(n=36)\end{array}$ & $\begin{array}{l}\text { Unexposed } \\
(n=36)\end{array}$ & $Z$ value & $p$ value \\
\hline Peg-board dominant & $68(14)$ & $65.5(14 \cdot 5)$ & 0.95 & $0 \cdot 34$ \\
\hline Peg-board non-dominant & $70(14 \cdot 5)$ & $69.5(17.5)$ & 0.39 & 0.69 \\
\hline Trail-making A & $39(21)$ & $35(17.5)$ & $1 \cdot 48$ & 0.13 \\
\hline Trail-making B & $108(45 \cdot 5)$ & $88 \cdot 5(57 \cdot 5)$ & 0.96 & 0.33 \\
\hline Digit symbol reproduction & $6(3 \cdot 5)$ & $7(3)$ & $1 \cdot 34$ & $0 \cdot 18$ \\
\hline Kimura recognition & 31 (12) & $33 \cdot 5(11)$ & 0.50 & 0.61 \\
\hline Paired associate learning & $29.5(21.5)$ & $19 \cdot 5(20)$ & $2 \cdot 81$ & 0.005 \\
\hline \multicolumn{5}{|l|}{ Continuous word recognition } \\
\hline Hits short interval & $3 \cdot 7(1 \cdot 7)$ & $4 \cdot 3(0 \cdot 7)$ & $2 \cdot 77$ & 0.006 \\
\hline Hits long interval & $4.0(1.7)$ & $4 \cdot 3(0 \cdot 8)$ & $2 \cdot 19$ & 0.028 \\
\hline Simple RT $(\mathrm{ms})^{\star}$ & $219.5(47)$ & $219.5(31)$ & 1.05 & $0 \cdot 29$ \\
\hline Complex RT (ms)* & $380 \cdot 5$ (49) & $372 \cdot 5(40)$ & $0 \cdot 84$ & 0.40 \\
\hline Hand-eye coordination & $1.7(0.5)$ & $1.7(0.5)$ & 0.90 & 0.36 \\
\hline POMS (sum of subscales) ${ }^{\star}$ & $47(10 \cdot 5)$ & $46 \cdot 5(8 \cdot 5)$ & 0.05 & 0.96 \\
\hline
\end{tabular}

${ }^{\star} \mathrm{RT}=$ Reaction time; POMS $=$ profile of mood states.

\section{Results}

The test variables showed distributions of varying degrees of normality and to use a consistent statistic, the Wilcoxon nonparametric two-sample test for non-independent samples is reported. Test results are consistently reported as raw scores, and all standardised tests were scored according to published standard procedures for scoring.

The groups were comparable on general measures of verbal and visuospatial function from the WAIS, but significant differences were observed for digit span and symbol digit substitution (table 1).

On neuropsychological and performance tests significant differences were found on number of trials before reaching criterion on paired associate learning (table 2), and for number of items correctly identified on continuous word recognition. Significant differences on computerised measures of reaction time or visuo-motor coordination were not found, and indications of mood differences were not found on the total score nor on any of the subscales.

A further analysis of subscales in tests showing significant findings is of interest as a basis for interpreting the results. Thus paired associate learning was analysed on the first recall attempt as well as on total trials to criterion and delayed recall. All measures showed significant group differences, but only for difficult items on the delayed recall measure. Continuous word recognition was categorised into performance on words with six or less intervening items $v$ words with 12 or more intervening items (see table 2 ).

\section{Discussion}

Group differences were found in several tests of memory and speed, whereas cognitively complex tasks measuring verbal and nonverbal abilities did not show significant differences. All the observed differences are in the direction of indicating poorer test results in the group of workers exposed to solvents, and may thus indicate a harmful effect of workrelated solvent exposure. The results thus confirm previous findings ${ }^{2-4}$ while avoiding the bias towards self-referred subjects in several reported studies. This study may also contain a selection bias: the healthy worker effect. If workers who have experienced symptoms have left their jobs and thus not been included in the study, this bias would decrease the likelihood of finding significant deficits in active workers. The fact that our study design is conservative tends to strengthen the findings.

The issue of controlling for pre-morbid ability differences in neurotoxicological research has been controversial. ${ }^{12}$ Tests of vocabulary and verbal conceptual reasoning may show deficits in some studies of workers exposed to solvents, ${ }^{1}$ and the differences may reflect selection factors to certain professions. In this study a test of verbal conceptual reasoning (definition of similarities) was chosen as a measure of verbal ability. This test is 
recommended in neuropsychology ${ }^{9}$ because the results are less dependent on schooling than tests of factual knowledge. As the groups in this study did not differ significantly on this test, it can be argued that the group selection criteria have functioned appropriately. There was a non-significant trend towards lower scores in the exposed group on Similarities, but repeated statistical analyses with score on Similarities as covariate resulted in only minor adjustments of the significance levels, and all major findings, including differences on paired associate learning, held up. The study by Morrow et $a l^{4}$ also reports a deficit in Paired Associate learning and Digit Span.

Performance measures that have been found to be sensitive in epidemiological research were included in addition to more traditional neuropsychological tests. We found no evidence that computerised tasks based on subtle reaction time measures, as in the NES 2 battery, have a higher sensitivity than neuropsychological tests.

Earlier reports ${ }^{13}$ indicate that subjective reports of neurasthenic symptoms are sensitive to mild toxic effects. In this study no differences in reported symptoms were found on mood scales corresponding closely to WHO standards. It could be argued that the significant findings on cognitive tests in our study are caused by acute effects. It is therefore worth noting that we did not observe group differences on other measures assumed to be more sensitive to acute effects.

Several memory tests in our study show significant deficits. The continuous recognition memory paradigm was included in the study to facilitate a theoretically motivated analysis of memory mechanisms. The results on this paradigm as well as the other memory tests may indicate that short- and long-term storage mechanisms are affected in the study group.

1 Arlien-Søborg A, Bruhn P, Gyldenstedt C, Melgaard B. Chronic painter's syndrome: chronic toxic encephalopathy in house painters. Acta Neurol Scand 1979;60: 149-59.

2 Hänninen $H$, Eskelinen L, Husman $K$, Nurminen $M$. Behavioral effects of long-term exposure to a mixture of organic solvents. Scand $\mathcal{f}$ Work Environ Health 1976;4: organic $240-55$.

3 Mikkelsen S, Jørgensen M, Browne E, Gyldensted C. Mixed solvent exposure and organic brain damage. A study of painters. Acta Neurol Scand Suppl 1988;78: study of

4 Morrow LA, Robin N, Hodgson MJ, Kamis $H$. Assessment of attention and memory efficiency in persons with solvent neurotoxicity. Neuropsychologia 1992; 30:911-22.

5 Iregren A, Letz R. Computerized testing in neurobehavioral toxicology. Appl Psychol Int Rev 1992;41:247-55.

6 Iregren A, Gamberale F. Human behavioral toxicology. Central nervous effects of low-dose exposure to neurotoxic substances in the work environment. Scand $\mathcal{F}$ Work Environ Health 1990;16(suppl 1):17-25.

7 Johnson BL, ed. Prevention of neurotoxic illness in working populations. New York: Wiley, 1987

8 Ryan CM, Morrow LA, Bromet EJ, Parkinson DK. Assessment of neuropsychological dysfunction in the Assessment of neuropsychological dysfunction in the Workplace; normative data from the Pittsburgh Occupational Exposure Test
Neuropsychol 1987;9:665-79.

9 Lezak M. Neuropsychological assessment. 2nd edn. New York: Oxford University Press, 1983.

10 Poon LW, Fozard JL. Age and word frequency effects in continuous recognition memory. F Gerontol 1980;35: $77-86$

11 Letz R, Baker EL. NES 2-neurobehavioral evaluation system. Winchester MA: Neurobehavioral Systems Inc. 1988.

12 Gade A, Mortensen EL, Bruhn P. "Chronic painter's syndrome". A reanalysis of psychological test data in a group of diagnosed cases based on comparison with group of diagnosed cases based on comparison with

13 Baker EL, Seppäläinen AM Hcand 1988;77:293-306. neurobehavioral effects. Neurotoxicology 1986;7:43-56. 